\title{
Recent Advances of Ultrasonography in Ruminants
}

\author{
Mohsin Ali Gazi* \\ Department of Animal husbandry, India \\ *Corresponding author: Mohsin Ali Gazi, Department of Surgery and Radiology, Jammu and Kashmir, India

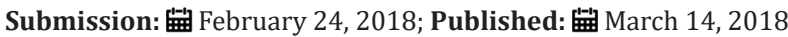

\section{Opinion}

Ultrasonography (USG) is not a new technology in veterinary medicine, since it has been utilized in various fields for more than twenty years. USG is a well established, non invasive, diagnostic imaging technique that provides unique information about the structure of soft tissues \& enables the evaluation of the motion pattern of certain organs \& structures. In veterinary practise it started with detection of ovine pregnancy in 1966. Since then, refinements in equipment quality combined with an increased awareness of the benefits of ultrasound as an imaging technique have led to its widespread use in the veterinary field.

In veterinary field, it has a widespread applicability from small to large animals in diagnosis \& therapeutics. Working in farm makes it mandatory to improve conception rate of cows \& increase productivity. Prolonged postpartum intervals to conception \& low conception rate, which are the results of inefficiencies in the management of nutrition, oestrus \& Artificial Insemination (AI) services, form the main constraints of cattle reproduction Reproductive efficiency can be enhanced by early identification of non-pregnant dairy cows \& heifers post breeding \& pregnancy rate by decreasing the interval between AI services \& increasing AI service rate. Thus, new technologies to pinpoint non-pregnant dairy cows \& heifers early after AI may play a key role in management strategies to improve reproductive efficiency \& profitability. Rectal palpation could be an accurate method for diagnosing pregnancy but is a poor method for resolving ovarian follicular distinction. Farin et al. 2006 reported that differentiation between follicular \& luteal cysts via rectal palpation is difficult, even for experienced practitioners but accuracy of diagnosis increases when using transrectal ultrasonography.

With the help of ultrasound Pregnancy diagnosis can be quickly \& precisely identified using as early as 26 days post (AI). Reports are also available about the detection of an embryonic vesicle in cattle as early as 9 day of gestation. However, to ensure fetus viability, scanning at 30 days pregnancy is necessary.

Benoit et.al 2004, documented that at approximately day 50 of gestation, male \& female fetuses (Fetal sexing) can be differentiated by the relative location of the genital tubercle \& development of the genital swellings into the scrotum in male foetuses. Additionally, the profitability of an ultrasound can be maximized through the scanning of other regions to accurately diagnose \& properly apply treatments to infections \& damages of the liver, bladder, mammary gland, lungs, \& kidneys, as well as identifying musculoskeletal \& viscera. On USG various cysts, hematomas, or abscesses can be very well differentiated. It also allows the visualization of the mammary gland (teat \& parenchyma) \& their morphological changes.

It is nowadays possible that even Eye examination with ultrasound for stephylomas, retinal detachments, foreign objects, cataracts, tumors, glaucoma, anterior chamber pathology such as hypopion, \& many other vision-related problems can be taken care. Cervical ultrasonography, especially for evaluating the thyroid lobes, is another common veteri application. Ultrasonography is an excellent tool for safely \& accurately guiding a needle into a nodule or mass to ensure proper sampling \& an accurate diagnosis. In addition, the use of USG to guide Abdominocentesis, Thoracocentesis, Pericardiocentesis, Cystocentesis, or biliary centesis increases the safety of each procedure when small volumes of fluid are sampled.

More over Ultrasound can be used to gain valuable information about the urogenital system. The bladder, ureters, \& urethra can be examined for stones, thickening of the lumen walls, or mural lesions of the bladder. It is a non-invasive method for diagnosis of urolithiasis, localization of urethral calculi \& rupture of urethra or the urinary bladder Gazi et al. 2013. USG has undoubtly resulted in an improved success rate of urolithiasis because of being earliest mode of detection \& diagnosis in small ruminants.

Additionally open field grazing make our livestock prone to ingestion of some foreign materials like polythene, nails, pins, balls, wood pieces \& other leading to foreign body syndrome followed by congestive heart failures \& finally death if not treated on time. Radiopaque objects can be located by Conventional radiographs but radiolucent bodies like wooden splinters are difficult to detect $\&$ are usually missed. The missed foreign bodies may produce immediate symptoms like wound infections or may remain 
asymptomatic for even decades. USG is a highly sensitive \& accurate modality in detecting radiolucent foreign bodies that are difficult to be visualized on standard radiographs (Figure 1).

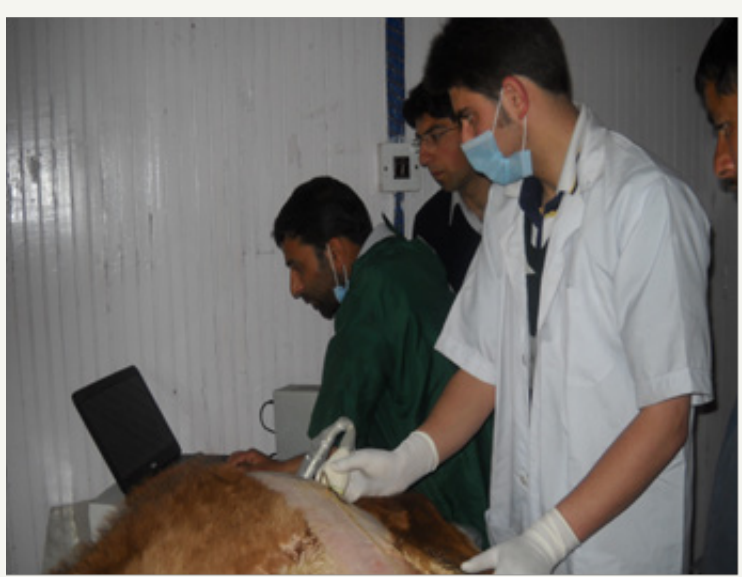

Figure 1: Ultrasonography of a calf being undertaken.
In the end Ultrasonography is an easy applicable, modern \& noninvasive method, allowing the visualization of the various organs \& their morphological changes. Also the largest advantages of sonography are a relatively simple \& effective use, safe to the subject \& the operator, its portability \& fast interpretation \& diagnosis in most circumstances.
Creative Commons Attribution 4.0 International License

For possible submissions Click Here



Approaches in Poultry, Dairy \& Veterinary Sciences

\section{Benefits of Publishing with us}

- High-level peer review and editorial services

- Freely accessible online immediately upon publication

- Authors retain the copyright to their work

- Licensing it under a Creative Commons license

- Visibility through different online platforms 